\title{
Non-coplanar whole brain radiotherapy is an effective modality for parotid sparing
}

\author{
Jaehyeon Park ${ }^{1}$, Jae Won Park ${ }^{2}$, Ji Woon Yea ${ }^{2}$ \\ ${ }^{1}$ Department of Radiation Oncology, Chonbuk National University Hospital, Chonbuk National University Medical School, Jeonju, Korea \\ ${ }^{2}$ Department of Radiation Oncology, Yeungnam University College of Medicine, Daegu, Korea
}

Received: November 16, 2018

Revised: December 25, 2018

Accepted: December 26, 2018

Corresponding author:

Jae Won Park

Department of Radiation Oncology,

Yeungnam University College of

Medicine, 170, Hyeonchung-ro,

Nam-gu, Daegu 42415, Korea

Tel: +82-53-620-3371

Fax: +82-53-624- 3599

E-mail:kapicap@ynu.ac.kr
Background: The purpose of this study was to evaluate the efficacy and feasibility of non- coplanar whole brain radiotherapy (NC-WBRT) for parotid sparing.

Methods: Fifteen cases, previously treated with WBRT were selected. NC-WBRT plans were generated. The beam arrangement for the non-coplanar plans consisted of superior anterior, right, and left beams. After generation of the non-coplanar plans a field-in-field technique was applied to the bilateral parallel opposed beams in order to reduce maximum dose and increase dose homogeneity. The NC-WBRT plans were subsequently compared with the previously generated bilateral WBRT (B-WBRT) plans. A field-in-field technique was also used with the B-WBRT plans according to our departmental protocol. As per our institutional practice a total dose of $30 \mathrm{~Gy}$ in 10 fractions of WBRT was administered 5 days a week.

Results: The mean dose to the parotid gland for the two different plans were 16.2 Gy with BWBRT and 13.7 Gy with NC-WBRT ( $p<0.05)$. In the NC-WBRT plan, the V5Gy, V10Gy, V15Gy, V20Gy, and V25Gy of the parotid were significantly lower $(p<0.05)$ than those of the B-WBRT plan. The $D_{\max }$ of the lens was also lower by $10 \%$ with NC-WBRT.

Conclusion: The use of NC-WBRT plans could be a simple and effective method to reduce irradiated volumes and improve the dose-volume parameters of the parotid gland.

Keywords: Organ at risk; Parotid gland; Whole brain radiotherapy; Xerostomia

\section{Introduction}

Whole brain radiotherapy (WBRT) is widely used with palliative intent in patients with multiple brain metastases and with prophylactic intent in patients with small cell lung cancer having good responses after primary treatment [1-7]. Issues related to WBRT-associated toxicities have been mostly neglected because the outcomes of patients requiring WBRT are generally poor. However, owing to advances in cancer therapy, survival times are much longer, and the issue of quality of life has presently gained importance [8].

The WBRT technique which employs parallel opposed fields is a simple and effective method to encompass the whole brain. During WBRT the main organs at risk are considered to be the lenses and the aero-digestive tract because these organs are particularly radiosensitive and irradiation increases the risk of cataracts and dysphagia $[9,10]$.

Although there was not much change in target coverage in the transition from 2-dimensional radiation therapy (2D-RT) to 3-dimensional conformal radiation therapy (3D-CRT), computed tomography (CT)-based simulation made it possible to evaluate the dose distribution in the normal organs. This advantage also made it possible to discover that large volumes of the parotid gland were being irradiated to clinically meaningful

Copyright@ 2019 Yeungnam University College of Medicine

This is an Open Access article distributed under the terms of the Creative Commons Attribution Non-Commercial License (http://creativecommons.org/licenses/by-nc/4.0/) which permits unrestricted non-commercial use, distribution, and reproduction in any medium, provided the original work is properly cited. 
doses during WBRT. To avoid severe xerostomia, the Quantitative Analyses of Normal Tissue Effects in the Clinic (QUANTEC) guidelines recommend that the mean dose to at least one parotid gland should be less than 20 Gy or that the mean dose to the combined volume of both glands should be less than $25 \mathrm{~Gy}$ [11]. However Trignani et al. showed that $28 \%$ of patients treated with conventional treatment received parotid gland doses beyond the recommended limits [12]. Noh et al. have recently showed through the normal tissue complication probability model that the parotid gland should be considered an organ-at-risk (OAR) during WBRT [13].

Several institutions have tried to reduce the dose to the parotid gland usually by modifying the lower margin of the radiotherapy field and by using intensity-modulated radiation therapy (IMRT). Modifying the field has shown good results in sparing the parotid glands but uncertainty in target coverage remains an issue [14]. IMRT is also an excellent method to spare the parotid gland but it is not cost-effective [15]. We speculated that the superior anterior beam could effectively block the parotid gland without compromising target coverage. In this study, we evaluated the efficacy and feasibility of non-coplanar WBRT by adding a superior anterior beam.

\section{Materials and methods}

Fifteen patients with brain metastases previously treated with WBRT were selected. The median age of this cohort was 59 years and lung cancer was found to be the most common primary tumor. The patient characteristics of this cohort are summarized in Table 1 . In all these patients CT simulation was performed in the supine position using a thermoplastic mask for immobilization. We obtained CT scan images of $5 \mathrm{~mm}$ slice thickness and contoured the OARs, including both parotid glands and both the lenses. The brain contours were identified by autosegmentation. The clinical target volume (CTV) included the brain parenchyma and the spinal cord up to the lower level of the atlas. The CTV was expanded by $7 \mathrm{~mm}$ in all directions to create the planning target volume (PTV).

The non-coplanar WBRT (NC-WBRT) plans were then generated. The beam arrangement for the non-coplanar plan consisted of superior anterior, right, and left beams. After generation of the non-coplanar plan, a field-in-field technique was applied to the bilateral parallel opposed beams for reducing the maximum dose and to increase dose homogeneity (Fig. 1). The NC-WBRT plans were subsequently compared with the previously generated bilateral WBRT (B-WBRT) plans. The field-in-field technique was also used with the B-WBRT plans according to our departmental protocol. According to our institutional practice WBRT is administered using the schedule of $30 \mathrm{~Gy}$ in 10 fractions for 5 days a week.

The conformity index (CI) and homogeneity index (HI) were utilized in treatment plan analysis. The $\mathrm{CI}$ is defined as the ratio of the volume of PTV that receives $95 \%$ of the prescribed dose to the entire PTV while the $\mathrm{HI}$ is the ratio of the maximum target dose to the prescribed dose. The paired $t$ - and Wilcoxon signed rank-tests were used to compare the dosimetric outcomes including dose coverage and OAR doses between the $\mathrm{NC}-$ and $\mathrm{B}-$ WBRT plans. All the statistical analyses were performed using the IBM SPSS version 21.0 (IBM Co., Armonk, NY, USA).

Table 1. Patient characteristics

\begin{tabular}{lc}
\hline Variable & No. of patients \\
\hline Age (yr) & 59 \\
Median & $47-84$ \\
Range & \\
Sex & 9 \\
$\quad$ Male & 6 \\
$\quad$ Female & \\
Primary sites & 7 \\
$\quad$ Lung & 4 \\
Breast & 1 \\
Tonsil & 1 \\
Stomach & 1 \\
Colon & 1 \\
Ovary & \\
\hline
\end{tabular}

\section{Results}

The CI for the B- and NC-WBRT plans were $0.80 \pm 0.06$ and $0.84 \pm 0.06$, respectively. The $\mathrm{HI}$ was similar in both plans at a value of $1.04 \pm 0.01$. The dose-volume statistics of the parotid glands are summarized in Table 2 and the dose-volume histogram is shown in Fig. 2. Compared with the B-WBRT plans, the NCWBRT plans lowered the mean dose of the right and left parotid glands from 15.6 Gy to 13.9 Gy and from 15.9 Gy to $13.6 \mathrm{~Gy}$, respectively $(p<0.05)$. The V5, V10, V15, V20, and V25 for the combined volume of both parotid glands were also significantly lower in the NC-WBRT plans $(p<0.05)$. The parotid doses delivered by the NC-WBRT plans were significantly lower in terms of all dosimetric parameters except for the V5 and $\mathrm{D}_{\min }$. The greatest reduction was seen with respect to V25 which showed a difference of $19 \%$ in the right and $20 \%$ in the left parotid gland, respectively (Fig. 3). The $\mathrm{D}_{\max }$ of the lens was $6.6 \pm 1.7 \mathrm{~Gy}$ vs. 6.1 \pm 1.7 Gy on the right and $5.9 \pm 1.4$ Gy vs. $5.4 \pm 1.3 \mathrm{~Gy}$ on the left in the in the B-WBRT and NC-WBRT plans, respectively. 

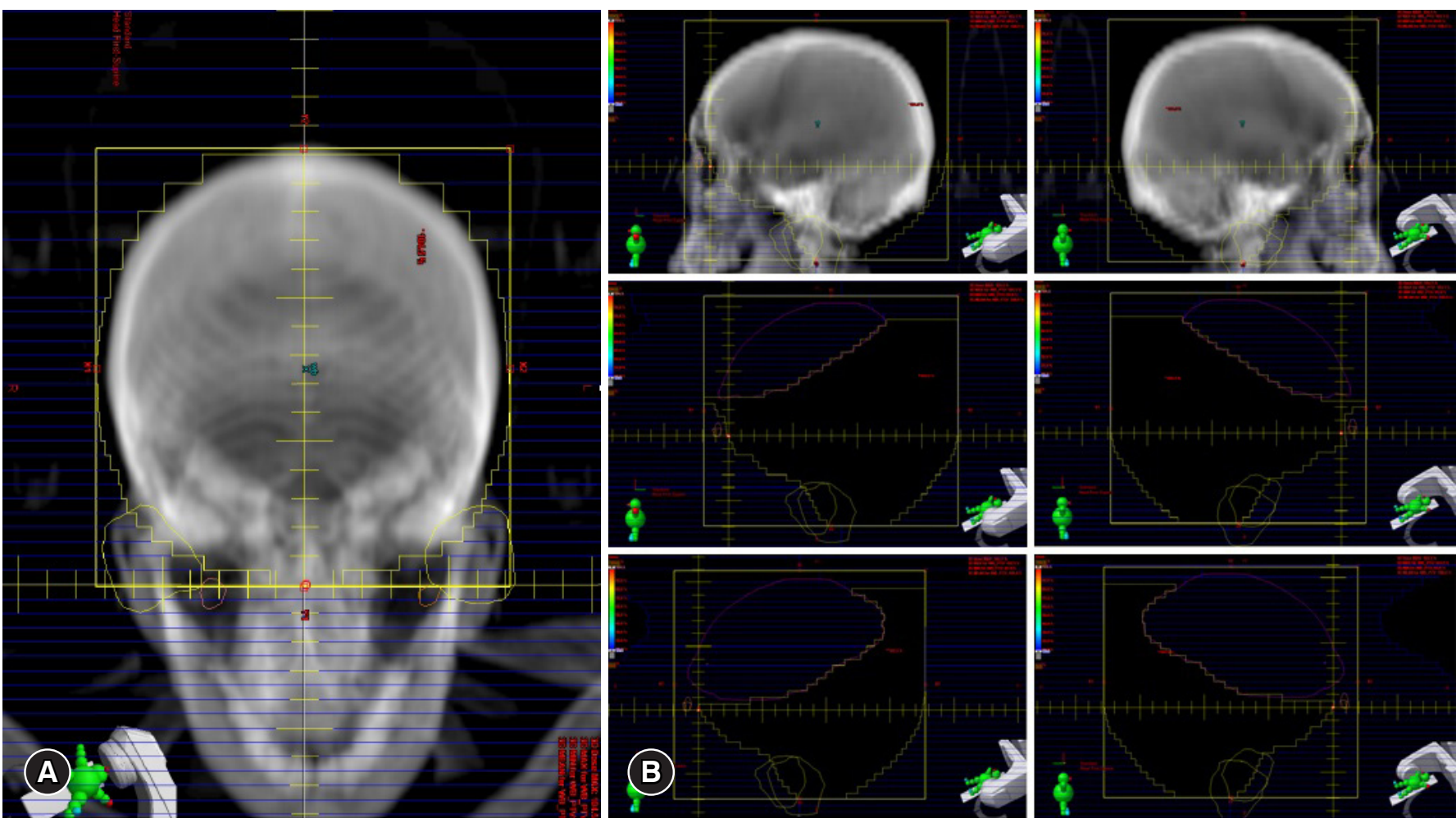

Fig. 1. Beam's eye view. (A) Superior anterior field of the NC-WBRT. (B) Field-in-field of B-WBRT. NC-WBRT, non-coplanar whole brain radiotherapy; B-WBRT, bilateral whole brain radiotherapy.

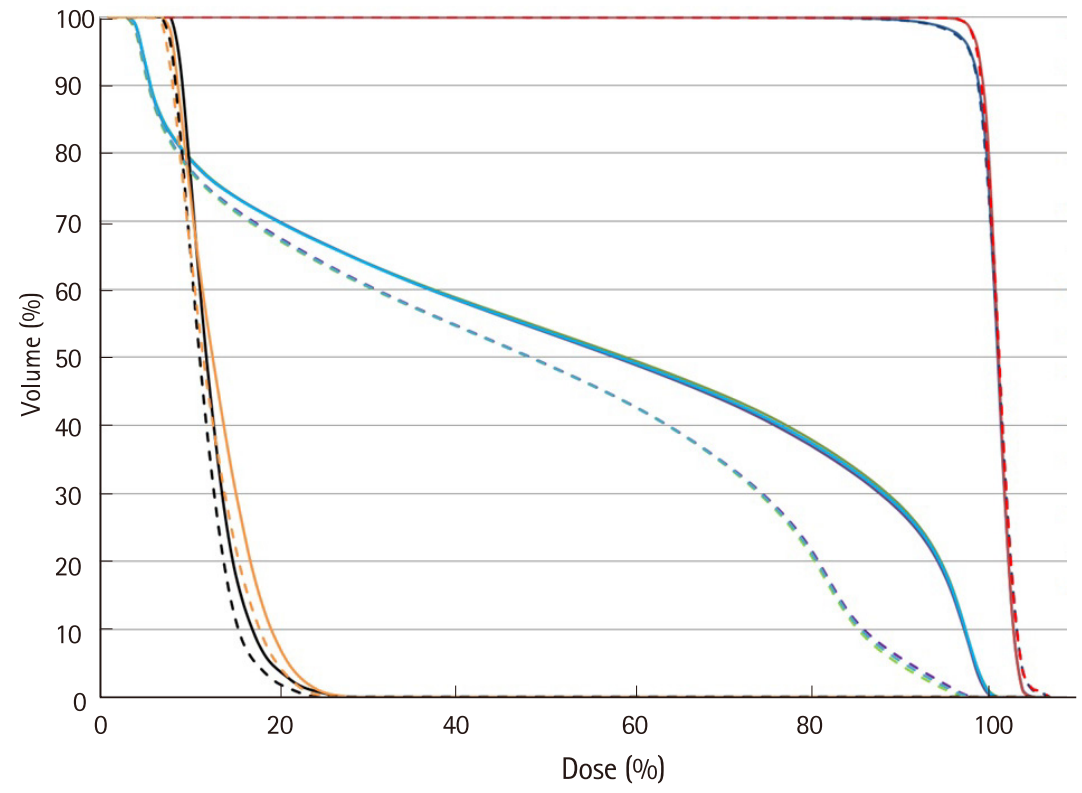

\section{- B-WBRT PTV}

— B-WBRT CTV

- B-WBRT parotid It.

- B-WBRT parotid rt.

- B-WBRT parotid both

- B-WBRT lens It.

- B-WBRT lens rt.

- - NC-WBRT PTV

- - NC-WBRT CTV

- - NC-WBRT parotid It.

- - NC-WBRT parotid rt.

- - NC-WBRT parotid both

- - NC-WBRT lens It.

- - NC-WBRT lens rt.

Fig. 2. Dose-volume histogram of parotid gland for B-WBRT and NC-WBRT. B-WBRT, bilateral whole brain radiotherapy; NC-WBRT, non-coplanar whole brain radiotherapy. PTV, planning target volume; CTV, clinical target volume; It., left; rt., right. 
Table 2. Dose-volume statistics of parotid gland with B-WBRT and NC-WBRT

\begin{tabular}{|c|c|c|c|c|c|c|}
\hline \multirow{2}{*}{ Volume } & \multirow{2}{*}{ Dose } & \multicolumn{2}{|c|}{ B-WBRT } & \multicolumn{2}{|c|}{ NC-WBRT } & \multirow{2}{*}{$p$-value } \\
\hline & & Median & SD & Median & SD & \\
\hline \multirow[t]{8}{*}{ Parotid rt. } & V5 (\%) & 71.2 & 11.1 & 70.4 & 11.6 & 0.009 \\
\hline & V10 (\%) & 61.0 & 11.5 & 53.9 & 12.2 & 0.001 \\
\hline & V15 (\%) & 53.0 & 9.7 & 49.4 & 10.3 & 0.001 \\
\hline & V20 (\%) & 44.1 & 10.6 & 36.7 & 9.5 & 0.001 \\
\hline & V25 (\%) & 33.2 & 9.2 & 14.0 & 5.5 & 0.001 \\
\hline & $\mathrm{D}_{\min }(\mathrm{cGy})$ & 86.5 & 21.4 & 88.5 & 18.5 & 0.012 \\
\hline & $\mathrm{D}_{\max }(\mathrm{cG} y)$ & $2,965.4$ & 31.4 & $2,919.0$ & 88.6 & 0.011 \\
\hline & $\mathrm{D}_{\text {mean }}(\mathrm{cGy})$ & $1,554.9$ & 285.3 & 1,389.5 & 241.4 & 0.001 \\
\hline \multirow[t]{8}{*}{ Parotid It. } & V5 (\%) & 72.8 & 10.6 & 70.3 & 10.7 & 0.008 \\
\hline & V10 (\%) & 62.8 & 11.1 & 55.2 & 11.1 & 0.001 \\
\hline & V15 (\%) & 56.1 & 12.1 & 50.0 & 11.3 & 0.001 \\
\hline & V20 (\%) & 46.0 & 10.4 & 36.0 & 9.9 & 0.001 \\
\hline & V25 (\%) & 32.9 & 9.8 & 12.3 & 6.2 & 0.001 \\
\hline & $\mathrm{D}_{\min }(\mathrm{cGy})$ & 92.6 & 21.4 & 85.3 & 18.4 & 0.001 \\
\hline & $\mathrm{D}_{\max }$ (cGy) & $2,964.8$ & 27.6 & $2,922.8$ & 66.5 & 0.002 \\
\hline & $\mathrm{D}_{\text {mean }}(\mathrm{cGy})$ & $1,593.3$ & 284.6 & $1,357.6$ & 235.8 & 0.001 \\
\hline \multirow[t]{8}{*}{ Parotid both } & V5 (\%) & 73.7 & 9.7 & 72.2 & 10.2 & 0.256 \\
\hline & V10 (\%) & 63.9 & 10.3 & 61.0 & 10.4 & 0.027 \\
\hline & V15 (\%) & 56.1 & 9.7 & 51.9 & 9.5 & 0.006 \\
\hline & V20 (\%) & 47.3 & 9.6 & 36.4 & 8.9 & 0.001 \\
\hline & V25 (\%) & 33.5 & 8.6 & 14.6 & 5.3 & 0.001 \\
\hline & $\mathrm{D}_{\min }(\mathrm{cGy})$ & 85.0 & 17.7 & 81.3 & 17.5 & 0.122 \\
\hline & $\mathrm{D}_{\max }(\mathrm{cGy})$ & 2,978.8 & 26.6 & 2,925.8 & 61.4 & 0.005 \\
\hline & $\mathrm{D}_{\text {mean }}(\mathrm{cGy})$ & $1,618.4$ & 261.9 & $1,372.4$ & 230.7 & 0.001 \\
\hline
\end{tabular}

B-WBRT, bilateral whole brain radiotherapy; NC-WBRT, non-coplanar whole brain radiotherapy; SD, standard deviation; rt., right; It., left.

\section{Discussion}

The incidence of brain metastases from all cancers is 5.0 per 1,000 person-years. The major primary sites in South Korea in the order of incidence are the lung, the liver, the breast, and the colorectum [16]. WBRT is the standard palliative treatment in most patients with multiple brain metastases. It is also employed as curative treatment in cases of primary central nervous system lymphoma, and as prophylactic therapy for small cell lung cancer with showing good responses to chemotherapy $[3,17,18]$.

The 'helmet' technique is usually employed because it provides good target coverage. This method consists of bilateral parallel opposed fields, which encompass the brain tissue, the skull, and the spinal cord to the lower level of the atlas. In the $2 \mathrm{D}$ era, the lens and the aero-digestive tract were considered the organs at risk. However, after the transition to the 3D era, by virtue of CT-simulation, it was possible to identify normal tissue dose distributions. Trignani et al. compared the $2 \mathrm{D}$ technique with the 3D technique for WBRT, and discovered that $28 \%$ of patients received a mean dose in excess of 20 Gy to the parotid gland when using 2D-RT [12]. Noh et al. also reported that mean parotid doses in 64 individual cases were in excess of $20 \mathrm{~Gy}$ and $25 \mathrm{~Gy}$ in $34.4 \%$ and $6.3 \%$, respectively [13]. In view of the recommended dose limits of the parotid gland by the QUANTEC guidelines [11], they concluded that the parotid glands could be regarded as organs-at-risk during WBRT. Burlage et al. have reported that salivary flow rates drop dramatically during the first 2 weeks of RT. In their study cohort, when the total cumulative dose was $20 \mathrm{~Gy}$, the flow rate had decreased by about $80 \%$ of the initial flow [19]. Considering that the most common treatment schedule of WBRT is $30 \mathrm{~Gy}$ in 10 fractions, the possibility of parotid gland dysfunction after WBRT cannot be excluded.

Some efforts have been made to reduce higher doses to irradiated parotid volumes. Most of these attempted to achieve this by adjusting the radiation fields. Fiorentino et al. reported on the dosimetric data of the parotid gland for 30 patients who received WBRT [20]. The median V20 and V25 of the right parotid gland were $3.5 \%$ (range, $0-44.5 \%$ ), and $1.85 \%$ (range, 0.3-18.0\%), respectively. For the left parotid gland, median V20 and V25 were $3.1 \%$ (range, $0-44.5 \%$ ) and $1.8 \%$ (range, $0-32.2 \%)$, respectively. They had only revised WBRT with 

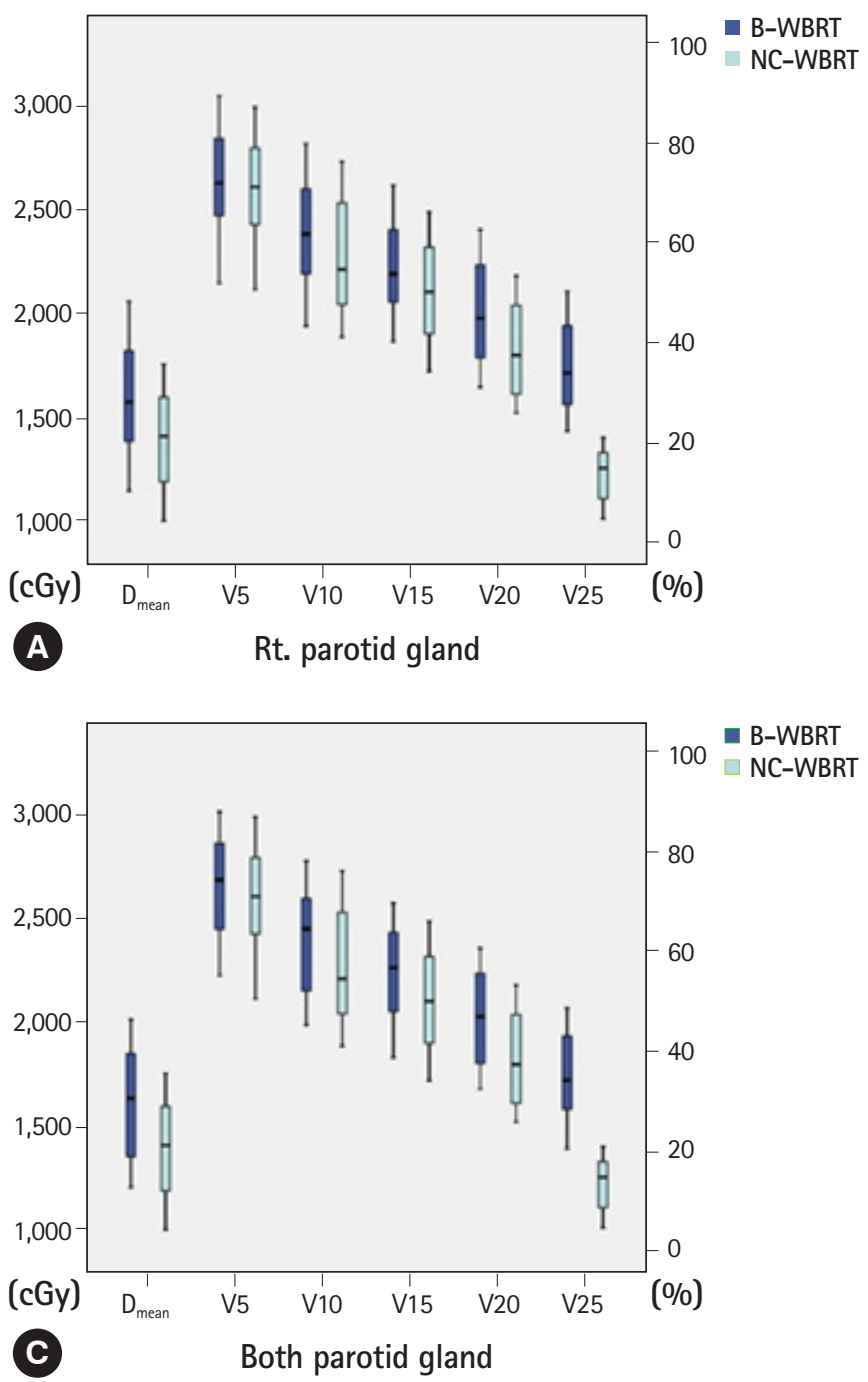

the treatment fields encompassing the brain tissue, and the skull. Compared to our results, the volume of parotid gland irradiated to higher doses in their study was very low. Cho et al. have shown that a modified field (MF) customized for the brain tissue is an effective technique for sparing the parotid gland, compared with conventional fields (CF) [14]. The mean parotid doses with $\mathrm{CF}$ and MF were $17.4 \mathrm{~Gy}$ and $8.7 \mathrm{~Gy}$, respectively. The V20 of both plans were $48.4 \%$ and $18.2 \%$, respectively. In our study, the median parotid dose was 13.7 Gy and V20 was 36.4\% in NC-WBRT, which was better than that of CF. However, although parotid sparing was better with the MF technique, there have been some concerns about target coverage with the MF because the radiation field is restricted to the parotid gland region. Cho et al. also reported that target coverage with MF was statistically lower than that of CF although this was not clinically significant [14].

All techniques used in the studies mentioned above including our study met the dose constraints of the parotid gland as per

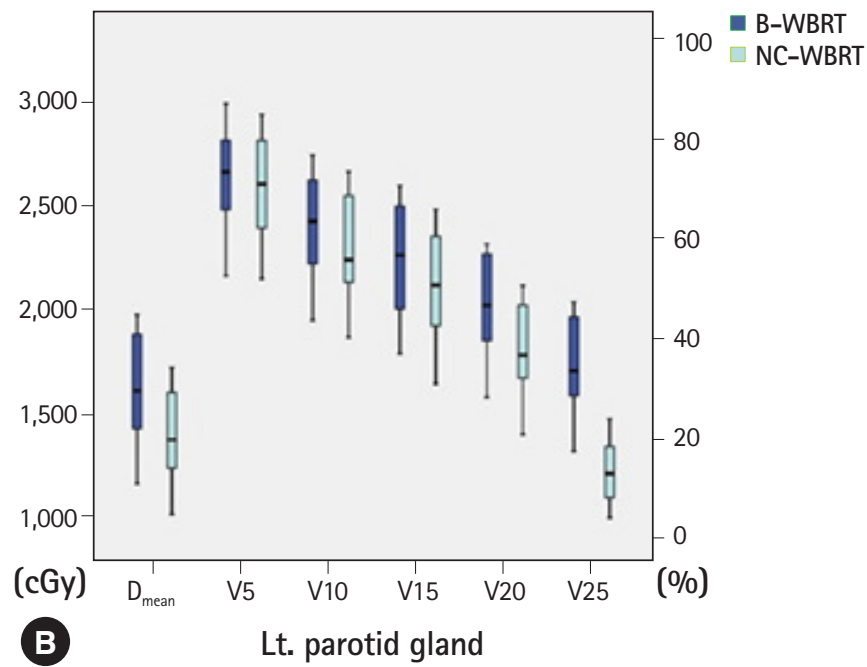

Fig. 3. Boxplot of $D_{\text {mean }}$ and $V 5-V 25$ for (A) right, (B) left, and (C) both parotid gland. B-WBRT, bilateral whole brain radiotherapy; NC-WBRT, non-coplanar whole brain radiotherapy.

the QUANTEC recommendations. This guideline is based on the relationship of the incidence of severe xerostomia with the dose-volume parameters of the parotid. Deasy et al. in their study, found that at doses below the range of 10 to $15 \mathrm{~Gy}$ the reduction of parotid function was minimal but this gradually increased, when the received doses were in the range of 20 to 40 Gy [11]. The reduction in function of the parotid gland could lead to problems such as poor dental hygiene, oral infections and difficulty in chewing, and swallowing [21]. These problems contribute to a poor quality of life. It is therefore important to reduce doses to the parotid gland as far as possible for better salivary flow.

IMRT which provides improved dose homogeneity and conformity, is a useful technique for lowering the dose in complex-shaped organs; studies on hippocampus-sparing WBRT using IMRT, have recently been performed $[15,22,23]$. The efficacy of IMRT in sparing the parotid gland has already been demonstrated in head and neck cancers [24-26] and 
holds promise in WBRT. However, IMRT is nearly twice as expensive as $3 \mathrm{D}-\mathrm{CRT}$ and requires a longer preparation time. It is therefore not employed in all whole brain treatments, because most of the patients who undergo WBRT have a short life expectancy and the intent of therapy is essentially palliative. The rate of IMRT utilization in Korea has steadily increased since 2011 accounting for about $25 \%$ of all RT treatments in 2016. According to the Korean Health and Insurance Review and Assessment Service data, fewer IMRT treatments were noted in those areas that are geographically situated away from the state capital [27]. Owing to the limitations in IMRT services reduction of OAR-doses using 3D-CRT may be a more practical solution.

The lens is well-known for its radiosensitivity; small radiation doses may lead to cataract formation as a late complication. The recommended limit to the lens is around 5 Gy delivered in a fractionated schedule [28]. Our study showed that the NCWBRT plans had reduced the $\mathrm{D}_{\text {max }}$ of the lens by $10 \%$.

\section{Conclusion}

NC-WBRT could be an effective and simple alternative to BWBRT offering comparatively lower irradiated parotid volumes and improved lens dosimetry without compromising target coverage.

\section{Conflicts of interest}

No potential conflicts of interest relevant to this article were reported.

\section{ORCID}

Jaehyeon Park, https://orcid.org/0000-0003-2546-4808 Jae Won Park, https://orcid.org/0000-0003-1100-5301 Ji Woon Yea, https://orcid.org/0000-0002-7699-342X

\section{References}

1. Coia LR. The role of radiation therapy in the treatment of brain metastases. Int J Radiat Oncol Biol Phys 1992;23:229-38.

2. Agboola O, Benoit B, Cross P, Da Silva V, Esche B, Lesiuk H, et al. Prognostic factors derived from recursive partition analysis (RPA) of Radiation Therapy Oncology Group (RTOG) brain metastases trials applied to surgically resected and irradiated brain metastatic cases. Int J Radiat Oncol Biol Phys 1998;42:155-9.
3. Aupérin A, Arriagada R, Pignon JP, Le Péchoux C, Gregor A, Stephens RJ, et al. Prophylactic cranial irradiation for patients with small-cell lung cancer in complete remission. $\mathrm{N}$ Engl J Med 1999;341:476-84.

4. Gaspar LE, Scott C, Murray K, Curran W. Validation of the RTOG recursive partitioning analysis (RPA) classification for brain metastases. Int J Radiat Oncol Biol Phys 2000;47:1001-6.

5. Shaw EG, Su JQ, Eagan RT, Jett JR, Maksymiuk AW, Deigert FA. Prophylactic cranial irradiation in complete responders with small-cell lung cancer: analysis of the Mayo Clinic and North Central Cancer Treatment Group data bases. J Clin Oncol 1994;12:2327-32.

6. Zhang W, Jiang W, Luan L, Wang L, Zheng X, Wang G. Prophylactic cranial irradiation for patients with small-cell lung cancer: a systematic review of the literature with meta-analysis. BMC Cancer 2014;14:793.

7. Bang A, Kendal WS, Laurie SA, Cook G, MacRae RM. Prophylactic cranial irradiation in extensive stage small cell lung cancer: outcomes at a comprehensive cancer centre. Int J Radiat Oncol Biol Phys 2018;101:1133-40.

8. Rohan EA, Miller N, Bonner F 3rd, Fultz-Butts K, PrattChapman ML, Alfano CM, et al. Comprehensive cancer control: promoting survivor health and wellness. Cancer Causes Control 2018;29:1277-85.

9. Poppe E. Experimental investigations on cataract formation following whole-body roentgen irradiation. Acta radiol 1957;47:138-48.

10. Rose-Ped AM, Bellm LA, Epstein JB, Trotti A, Gwede C, Fuchs HJ. Complications of radiation therapy for head and neck cancers. The patient's perspective. Cancer Nurs 2002;25:461-7.

11. Deasy JO, Moiseenko V, Marks L, Chao KS, Nam J, Eisbruch A. Radiotherapy dose-volume effects on salivary gland function. Int J Radiat Oncol Biol Phys 2010;76(3 Suppl): S58-63.

12. Trignani M, Genovesi D, Vinciguerra A, Di Pilla A, Augurio A, Di Tommaso M, et al. Parotid glands in whole-brain radiotherapy: $2 \mathrm{D}$ versus $3 \mathrm{D}$ technique for no sparing or sparing. Radiol Med 2015;120:324-8.

13. Noh OK, Chun M, Nam SS, Jang H, Jo S, Oh YT, et al. Parotid gland as a risk organ in whole brain radiotherapy. Radiother Oncol 2011;98:223-6.

14. Cho O, Chun M, Park SH, Oh YT, Kim MH, Park HJ, et al. Parotid gland sparing effect by computed tomography-based modified lower field margin in whole brain radiotherapy. Radiat Oncol J 2013;31:12-7.

15. Yu JB, Shiao SL, Knisely JP. A dosimetric evaluation of conventional helmet field irradiation versus two-field intensitymodulated radiotherapy technique. Int J Radiat Oncol Biol 
Phys 2007;68:621-31.

16. Kim T, Song C, Han JH, Kim IA, Kim YJ, Kim SH, et al. Epidemiology of intracranial metastases in Korea: a national cohort investigation. Cancer Res Treat 2018;50:164-74.

17. Bovi JA, Schultz CJ, Mehta MP, Corn BW. Consolidative whole-brain radiation therapy versus autologous stem cell transplant for primary central nervous system lymphoma: a large dose of perspective and perhaps a lower dose of radiation are in order. Int J Radiat Oncol Biol Phys 2018;102:59-60.

18. Slotman B, Faivre-Finn C, Kramer G, Rankin E, Snee M, Hatton M, et al. Prophylactic cranial irradiation in extensive small-cell lung cancer. N Engl J Med 2007;357:664-72.

19. Burlage FR, Coppes RP, Meertens H, Stokman MA, Vissink A. Parotid and submandibular/sublingual salivary flow during high dose radiotherapy. Radiother Oncol 2001;61:271-4.

20. Fiorentino A, Chiumento C, Caivano R, Cozzolino M, Clemente S, Pedicini P, et al. "Whole brain radiotherapy: are parotid glands organs at risk?". Radiother Oncol 2012;103:130-1.

21. Ship JA, Hu K. Radiotherapy-induced salivary dysfunction. Semin Oncol 2004;31(6 Suppl 18): 29-36.

22. Chung E, Noh JM, Lee KC, Kim JH, Chung W, Suh YG, et al. Dummy run of quality assurance program before prospective study of hippocampus-sparing whole-brain radiotherapy (HSWBRT) and simultaneous integrated boost (SIB) for multiple brain metastases from non-small cell lung cancer: Korean Radiation Oncology Group (KROG) 17-06 study. Cancer Res Treat 2018. doi: 10.4143/crt.2018.415 [Epub ahead of print].
23. Gondi V, Tolakanahalli R, Mehta MP, Tewatia D, Rowley H, Kuo JS, et al. Hippocampal-sparing whole-brain radiotherapy: a "how-to" technique using helical tomotherapy and linear accelerator-based intensity-modulated radiotherapy. Int J Radiat Oncol Biol Phys 2010;78:1244-52.

24. Gupta T, Agarwal J, Jain S, Phurailatpam R, Kannan S, GhoshLaskar S, et al. Three-dimensional conformal radiotherapy (3D-CRT) versus intensity modulated radiation therapy (IMRT) in squamous cell carcinoma of the head and neck: a randomized controlled trial. Radiother Oncol 2012;104:343-8.

25. Pow EH, Kwong DL, McMillan AS, Wong MC, Sham JS, Leung LH, et al. Xerostomia and quality of life after intensitymodulated radiotherapy vs. conventional radiotherapy for early-stage nasopharyngeal carcinoma: initial report on a randomized controlled clinical trial. Int J Radiat Oncol Biol Phys 2006;66:981-91.

26. Nutting CM, Morden JP, Harrington KJ, Urbano TG, Bhide SA, Clark C, et al. Parotid-sparing intensity modulated versus conventional radiotherapy in head and neck cancer (PARSPORT): a phase 3 multicentre randomised controlled trial. Lancet Oncol 2011;12:127-36.

27. Rim CH, Lee J, Kim WC, Yang D, Yoon WS, Koom WS, et al. A survey of radiation therapy utilization in Korea from 2010 to 2016: focusing on use of intensity-modulated radiation therapy. J Korean Med Sci 2018;33:e67.

28. Henk JM, Whitelocke RA, Warrington AP, Bessell EM. Radiation dose to the lens and cataract formation. Int J Radiat Oncol Biol Phys 1993;25:815-20. 